\section{(C) OPEN ACCESS}

\title{
Deep brain stimulation in the caudal zona incerta versus best medical treatment in patients with Parkinson's disease: a randomised blinded evaluation
}

\author{
Patric Blomstedt, ${ }^{1}$ Rasmus Stenmark Persson, ${ }^{1}$ Gun-Marie Hariz, ${ }^{1,2}$ Jan Linder, ${ }^{1}$ \\ Anna Fredricks, ${ }^{1}$ Björn Häggström, ${ }^{1}$ Johanna Philipsson, ${ }^{1}$ Lars Forsgren, ${ }^{1}$ \\ Marwan Hariz ${ }^{1,3}$
}

'Department of Pharmacology and Clinical Neuroscience, Umeå University, Umeå, Sweden 2Unit of Occupational Therapy, Department of Community Medicine and Rehabilitation, Umeå University, Umeå, Sweden ${ }^{3}$ Unit of Functional Neurosurgery, UCL Institute of Neurology, London, UK

\section{Correspondence to} Dr Patric Blomstedt, Department of Clinical Neuroscience, University Hospital of Umeå, Umeå 901 85, Sweden; patric. blomstedt@neuro.umu.se

LF and MH contributed equally.

Received 8 September 2017 Revised 29 November 2017 Accepted 9 January 2018 Published Online First 31 January 2018

\section{ABSTRACT \\ Background Several open-label studies have shown good effect of deep brain stimulation (DBS) in the caudal zona incerta (cZi) on tremor, including parkinsonian tremor, and in some cases also a benefit on akinesia and axial symptoms. The aim of this study was to evaluate objectively the effect of cZi DBS in patients with Parkinson's disease (PD). \\ Method 25 patients with PD were randomised to either cZi DBS or best medical treatment. The primary outcomes were differences between the groups in the motor scores of the Unified Parkinson's Disease Rating Scale (UPDRS- \\ III) rated single-blindly at 6 months and differences in the Parkinson's Disease Questionnaire 39 items (PDQ-39). 19 patients, 10 in the medical arm and 9 in the DBS arm, fulfilled the study.}

Results The DBS group had $41 \%$ better UPDRS-III scores off-medication on-stimulation compared with baseline, whereas the scores of the non-surgical patients off-medication were unchanged. In the on-medication condition, there were no differences between the groups, neither at baseline nor at 6 months. Subitems of the UPDRS-III showed a robust effect of CZi DBS on tremor. The PDQ-39 domains 'stigma' and 'ADL' improved only in the DBS group. The PDQ-39 summary index improved in both groups.

Conclusion This is the first randomised blinded evaluation of cZi DBS showing its efficacy on PD symptoms. The most striking effect was on tremor; however, the doses of dopaminergic medications could not be decreased. cZi DBS in PD may be an addition to existing established targets, enabling tailoring the surgery to the needs of the individual patient.

\section{INTRODUCTION}

Deep brain stimulation (DBS) is an established treatment for patients with Parkinson's disease (PD) when pharmacological therapy alone does not provide sufficient relief or is associated with disabling side effects. ${ }^{1}{ }^{2}$ At present, the subthalamic nucleus (STN) is the target of choice, but in some patients alternative targets, such as the ventral intermediate nucleus (VIM) of the thalamus or the globus pallidus internus (GPi), are preferred according to the symptomatic profile of PD or other patient-specific considerations. ${ }^{34}$ Recently, a number of studies have revived DBS in the posterior subthalamic area (PSA), a brain target that was commonly used for PD and tremors during the lesional era. ${ }^{5-9}$ The PSA is situated ventral to the VIM, between the red nucleus and the STN. This is a functionally crowded area that harbours the zona incerta including the caudal zona incerta (cZi), the Forel's fields, the lemniscus and the prelemniscal radiations, among many other structures and pathways. $^{1011}$

Open-label studies have reported good results of DBS in various parts of the subthalamic area for different forms of tremor, including parkinsonian tremor. ${ }^{12-20}$ Furthermore, none of the studies above had reported on psychiatric side effects that resemble what had been described following STN DBS, such as hypomania, apathy, depression or suicide. ${ }^{21-25}$ One intriguing study compared in a non-randomised unblinded manner DBS in the STN with DBS in $\mathrm{cZi}$ and suggested the latter to be more effective, not only for tremor, but also for rigidity and bradykinesia. ${ }^{16}$

Hence, it was of interest to evaluate in a more objective manner the effects of DBS of the $\mathrm{cZi}$ in patients with PD who otherwise fulfilled the criteria for STN DBS in order to establish its potential efficacy and its safety profile. The aim of the present work was thus to evaluate the effects of bilateral cZi DBS versus best medical treatment in a singleblinded randomised manner in a group of patients with PD who were eligible for bilateral STN DBS. The two primary outcomes were (a) differences in off-medication scores of the motor part of the Unified Parkinson's Disease Rating Scale (UPDRS-III) between medical and surgical groups at 6 months, and compared with their respective baseline scores, and (b) differences between the groups in quality of life rated on the 39 items of the Parkinson's Disease Questionnaire 39 items (PDQ-39).

\section{MATERIALS AND METHODS \\ Patients}

Patients who would normally be considered for bilateral STN DBS were eligible for participation in this study. All patients had previously undergone a ${ }^{123}$ I FP CIT SPECT to support the diagnosis of PD. The inclusion criteria were thus the same as for STN DBS, that is, idiopathic PD, as diagnosed by a senior movement disorders neurologist according to the criteria of the UK Parkinson's Disease Brain Bank ${ }^{26}$; 
L-dopa-responsive symptoms (defined as $>30 \%$ improvement in motor scores of the UPDRS-III), disabling motor fluctuations with or without dyskinesias or disabling tremor; age $<75$ years; no relevant psychiatric comorbidities or cognitive decline; normal brain MRI and absence of surgical contraindications. All patients underwent a formal neuropsychological evaluation, a standardised L-dopa challenge and a brain MRI to rule out atrophy or other contraindications for brain surgery. All patients were informed about the aim of the study and the possibility of having DBS in the 'established' STN target if they chose not to participate in the study. Informed consent was obtained according to the Declaration of Helsinki.

\section{Study design}

Patients were randomised to either bilateral cZi DBS within 1 month from inclusion or to best medical treatment (and, if needed, bilateral cZi DBS after 6 months). UPDRS-III on-medication/off-medication was administered at baseline and at 6 months on-medication/off-medication and on-stimulation/ off-stimulation by the same experienced evaluator (AF). The UPDRS-III scoring off-medication was done in the morning after withholding medication for 12 hours. The evaluation on-medication was done after administration of an L-dopa dose 50\% higher than the patient's normal morning dose. The evaluations at 6 months were conducted in both on-medication and off-medication states and on-stimulation/off-stimulation after the stimulator had been switched off/on for $60 \mathrm{~min}$.

All UPDRS-III evaluations were videotaped with the patients wearing head caps. The video documentations of UPDRS-III of each patient comprised 4-6 segments (baseline on-medication/ off-medication and 6 months on-medication/off-medication and on-stimulation/off-stimulation). The order of these segments was randomised and presented to two blinded experienced assessors (LF and $\mathrm{MH}$ ) who were not involved in selection of patients, surgery, or follow-up, and who were unaware of the patient's previous allocation. Two UPDRS items could not be evaluated on video: item 18 concerning speech and item 22 concerning rigidity. These two items from the unblinded UPDRS scoring were added to the blinded video-based scores of the motor UPDRS.

\section{Surgery}

The surgical procedure has been described in detail previously. ${ }^{27}$ In summary, stereotactic implantation of the DBS electrodes 3389 (Medtronic, Minneapolis, Minnesota, USA) was performed in local anaesthesia using the Leksell frame model G (Elekta Instruments, Linköping, Sweden). The brain target was identified anatomically on stereotactic thin-slice T2-weighted axial MR images. The target point lay slightly posteromedial to the visualised posterior tail of the STN on the scan showing the maximal diameter of the red nucleus (figure 1). An intraoperative stereotactic CT scan was performed with the frame in place and fused with the preoperative stereotactic MRI for verification of the electrode position (figure 2). If the position of the lead did not coincide with the intended target, the electrode was repositioned and a new CT scan performed. The implantable pulse generator (Kinetra/Activa PC, Medtronic) was implanted in the same surgical session. All procedures were performed by the same surgeon (PB). Microelectrode recording was not performed.

In the week after surgery, patients underwent in off-medication condition a systematic screening of electrode contacts to assess stimulation thresholds and effects on tremor,

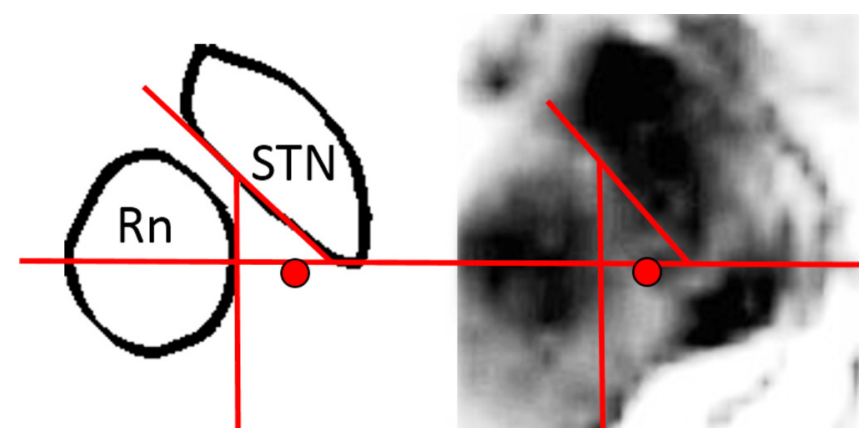

Figure 1 The target point in the caudal zona incerta, medial to the tail of the subthalamic nucleus (STN). Rn, red nucleus.

bradykinesia and rigidity, as well as stimulation-induced side effects. The contacts demonstrating the best effect in the absence of side effects were chosen for chronic stimulation. Stimulation settings and medications were evaluated and optimised during the study period, and eventual adverse events were recorded.

\section{Outcome measures}

The primary outcomes were the difference between groups at 6 months in the motor scores of the UPDRS-III and in health-related quality of life rated on the PDQ-39.

Secondary outcome measures included changes between baseline and 6 months in subscores of the UPDRS-III (tremor, speech, bradykinesia and axial subscores) in each group, as well as changes in dyskinesia and motor fluctuation scores according to UPDRS-IV, and in levodopa-equivalent daily doses (LEDD) between baseline and 6 months.

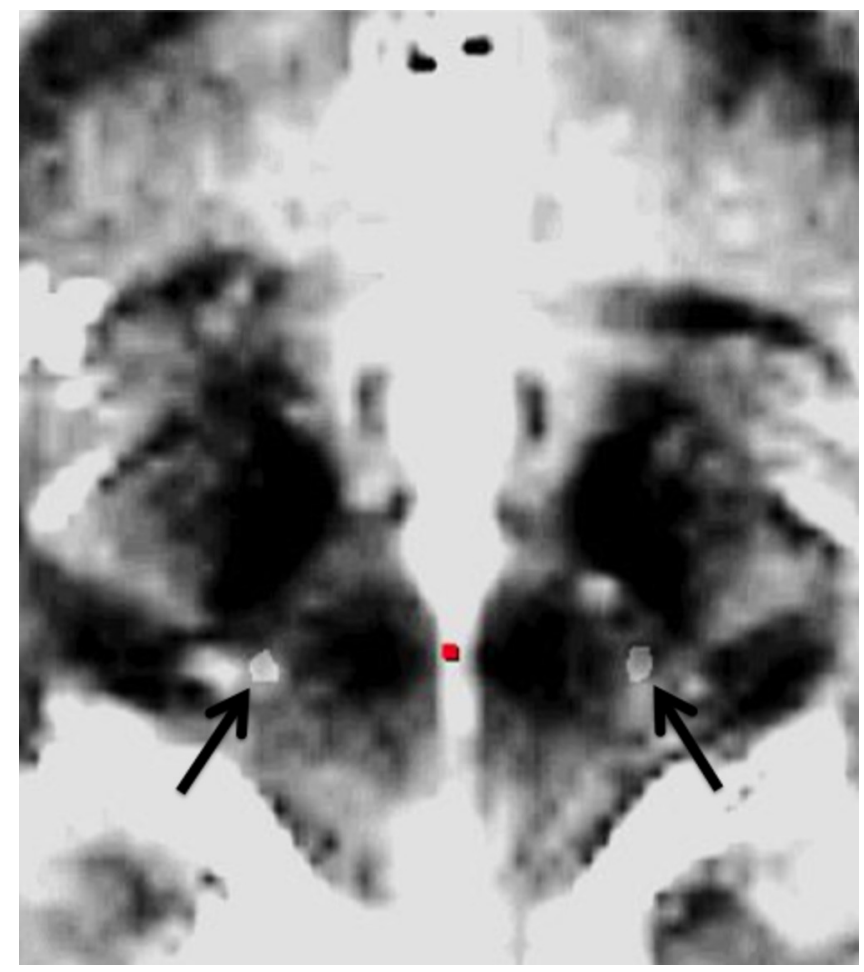

Figure 2 Stereotactic intraoperative CT scan fused with stereotactic preoperative T2-weighted MRI scan demonstrating bilateral electrodes (arrows) implanted in the caudal zona incerta. 
Table 1 Patients' characteristics in medical and surgical groups at baseline

\begin{tabular}{llll}
\hline & Medical group & Surgical group & P value \\
\hline Patients, $n$ & 10 & 9 & NS \\
\hline Male/female & $8 / 2$ & $7 / 2$ & NS \\
Age (years) & $60.9 \pm 9.2$ & $57 \pm 11.4$ & NS \\
Disease duration (years) & $10.3 \pm 5.6$ & $6.4 \pm 3$ & NS \\
\hline LEDD at baseline (mg) & $1043 \pm 516$ & $1376 \pm 883$ & $\mathrm{NS}$ \\
UPDRS-III at baseline off-medication & $42.4 \pm 14.5^{*}$ & $33.2 \pm 11.4^{* *}$ & $\mathrm{NS}$ \\
\hline UPDRS-III at baseline on-medication & $20.6 \pm 11.9^{*}$ & $19.4 \pm 12.5^{* *}$ & $\mathrm{NS}$ \\
\hline
\end{tabular}

Values represent mean \pm SD.

*Within-group, $\mathrm{P}=0.001$.

** Within-group, $P=0.01$.

LEDD, levodopa-equivalent daily doses; NS, not significant; UPDRS-III, Unified Parkinson's Disease Rating Scale.

\section{Statistics}

Patient demographic and clinical characteristics are presented as mean $\pm \mathrm{SD}$ and range. A two-tailed t-test was used for continuous variables. The Wilcoxon signed-rank test for paired samples was used for non-parametric data, to compare preoperative and postoperative scores within the medical and surgical group. Mann-Whitney $\mathrm{U}$ test was used for unpaired non-parametric data in order to compare scores between the medical and the surgical group. A P value of $<0.05$ was considered statistically significant.

\section{RESULTS}

Twenty-five patients met the inclusion criteria and accepted to be included in the trial. Of these 25 patients included and randomised, 6 could not or would not fulfil the trial: 4 patients (1 randomised to DBS and 3 to best medical management) quit directly after randomisation and did not wish to proceed. One patient randomised to DBS fell and broke her knee just before the surgery, and one patient refused to undergo evaluation with DBS turned off at 6 months. Hence, 19 patients (10 randomised to best medical management and 9 to DBS) fulfilled the trial and completed the evaluations at 6 months.

The patients' demographic and clinical characteristics at baseline are presented in table 1: there were no significant differences between the groups at baseline. Both groups showed good response to the L-dopa test preoperatively.

\section{Main outcomes}

Table 2 shows the scores of UPDRS-III between groups at 6 months. The scores were significantly better for surgical patients on-stimulation off-medication compared with medical patients

Table 2 Mean \pm SD of Unified Parkinson's Disease Rating Scale scores at 6 months in medical and surgical groups, respectively

\begin{tabular}{lll}
\hline & Off-medication & On-medication \\
\hline Medical group & $37.2 \pm 12.2$ & $21.8 \pm 13.4$ \\
Surgical group & & \\
Off-stimulation & $35.4 \pm 10.3$ & $20.3 \pm 81.6$ \\
On-stimulation & $19.5 \pm 7.8$ & $18.5 \pm 12.4$ \\
\hline
\end{tabular}

\section{P values:}

Medical group off-medication vs surgical group off-medication on-stimulation $\mathrm{P}=0.001$.

Medical group on-medication vs surgical group on-medication on-stimulation:

$\mathrm{P}=\mathrm{NS}$.

Within medical group: off-medication vs on-medication: $\mathrm{P}=0.001$.

Within surgical group: off-medication off-stimulation vs off-medication-onstimulation: $\mathrm{P}=0.001$ off-medication. In the on-medication condition, there were no significant differences in UPDRS scores between the two groups.

Table 3 shows the results of PDQ-39: the summary index was not different between medical and surgical groups, neither at baseline nor at 6 months. Compared with baseline, PDQ-39 summary index was improved in the surgical group by $36.4 \%$ and in the medical group by $24.4 \%$. In the surgical group, the improvement was significant concerning subdomains 'ADL' and 'stigma', and in the medical group the improvement concerned only the summary index of the PDQ-39. At baseline, as well as at 6 months, the surgical group scored better than the medical group on the dimension 'emotion'.

Table 3 Parkinson's Disease Questionnaire 39 items (PDQ-39) at baseline and at 6 months in medical and surgical groups, respectively

\begin{tabular}{|c|c|c|c|}
\hline PDQ-39 domains & $\begin{array}{l}\text { Medical group } \\
\text { Mean (SD) }\end{array}$ & $\begin{array}{l}\text { Surgical group } \\
\text { Mean (SD) }\end{array}$ & $P$ value \\
\hline Mobility baseline & $36.8(21.5)$ & $29.8(18.1)$ & NS \\
\hline Mobility 6 months & $28.5(24.5)$ & $20.6(16.0)$ & NS \\
\hline$P$ value & NS & NS & \\
\hline ADL baseline & $39.2(19.0)$ & $34.7(18.1)$ & NS \\
\hline ADL 6 months & $29.2(26.6)$ & $17.1(12.6)$ & NS \\
\hline$P$ value & NS & 0.028 & \\
\hline Emotion baseline & $30.8(16.2)$ & $13.4(9.7)$ & 0.022 \\
\hline Emotion 6 months & $24.5(15.4)$ & $8.8(6.7)$ & 0.022 \\
\hline$P$ value & NS & NS & \\
\hline Stigma baseline & $25.4(16.0)$ & $32.6(22.9)$ & NS \\
\hline Stigma 6 months & $18.2(11.6)$ & $9.7(9.4)$ & NS \\
\hline$P$ value & NS & 0.027 & \\
\hline Social baseline & $7.5(10)$ & $8.33(13.8)$ & NS \\
\hline Social 6 months & $7.5(12.1)$ & $0.92(2.8)$ & NS \\
\hline$P$ value & NS & 0.059 & \\
\hline Cognition baseline & $14.4(15.0)$ & $18.1(17.5)$ & NS \\
\hline Cognition 6 months & $12.5(12.5)$ & $13.2(14.1)$ & NS \\
\hline$P$ value & NS & NS & \\
\hline Communication baseline & $13.3(16.75)$ & $13.0(18.7)$ & NS \\
\hline Communication 6 months & $10.8(14.2)$ & $13.0(8.4)$ & NS \\
\hline$P$ value & NS & NS & \\
\hline Body baseline & $32.5(25.0)$ & $32.4(24.5)$ & NS \\
\hline Body 6 months & $21.7(18.5)$ & $20.4(17.2)$ & NS \\
\hline$P$ value & NS & NS & \\
\hline PDQ SI baseline & $25.0(8.3)$ & $22.8(13.2)$ & NS \\
\hline PDQ SI 6 months & $18.9(9.85)$ & $14.5(9.5)$ & NS \\
\hline$P$ value & 0.028 & 0.038 & \\
\hline
\end{tabular}

PDQ SI, Parkinson's Disease Questionnaire Summary Index; NS, not significant. 
Table 4 Mean \pm SD scores of selected items of Unified Parkinson's Disease Rating Scale (UPDRS-III), dyskinesia scores (UPDRS-IV) and levodopaequivalent daily doses (LEDD), at baseline and at 6 months in medical group on-medication/off-medication, and in surgical group on-medication/ off-medication on-stimulation/off-stimulation

\begin{tabular}{|c|c|c|c|c|c|c|c|c|c|c|}
\hline & \multicolumn{4}{|c|}{ Medical group } & \multicolumn{6}{|c|}{ Surgical group } \\
\hline & \multicolumn{2}{|l|}{ Baseline } & \multicolumn{2}{|l|}{6 months } & \multicolumn{2}{|l|}{ Baseline } & \multicolumn{4}{|l|}{6 months } \\
\hline & $\begin{array}{l}\text { Off- } \\
\text { medication }\end{array}$ & $\begin{array}{l}\text { On- } \\
\text { medication }\end{array}$ & $\begin{array}{l}\text { Off- } \\
\text { medication }\end{array}$ & $\begin{array}{l}\text { On- } \\
\text { medication }\end{array}$ & $\begin{array}{l}\text { Off- } \\
\text { medication }\end{array}$ & $\begin{array}{l}\text { On- } \\
\text { medication }\end{array}$ & $\begin{array}{l}\text { Off- } \\
\text { medication } \\
\text { off- } \\
\text { stimulation }\end{array}$ & $\begin{array}{l}\text { On- } \\
\text { medication } \\
\text { off- } \\
\text { stimulation }\end{array}$ & $\begin{array}{l}\text { Off- } \\
\text { medication } \\
\text { on- } \\
\text { stimulation }\end{array}$ & $\begin{array}{l}\text { On- } \\
\text { medication } \\
\text { on- } \\
\text { stimulation }\end{array}$ \\
\hline Tremor (items 20-21) & $8.3 \pm 6.4$ & $2.1 \pm 3.9$ & $6.9 \pm 5.6$ & $2.7 \pm 4.7$ & $5.1 \pm 3.4$ & $2.3 \pm 4.2$ & $6.1 \pm 4.6$ & $2.1 \pm 4.2$ & $0.4 \pm 0.5$ & $0.4 \pm 0.8$ \\
\hline Speech (item 18) & $1.3 \pm 0.7$ & $0.9 \pm 0.5$ & $1.2 \pm 0.8$ & $0.7 \pm 0.5$ & $1.2 \pm 0.7$ & $0.8 \pm 0.7$ & $1.3 \pm 0.7$ & $1 \pm 0.7$ & $1.2 \pm 0.7$ & $0.9 \pm 0.6$ \\
\hline Akinesia (items 22-26) & $15.8 \pm 5.6$ & $9.1 \pm 5.1$ & $14.4 \pm 4.4$ & $7.9 \pm 5.4$ & $14.3 \pm 6.6$ & $10 \pm 6.1$ & $15.7 \pm 6.3$ & $9.1 \pm 3.3$ & $9.7 \pm 5$ & $10.1 \pm 6.6$ \\
\hline Axial (items 27-31) & $4.4 \pm 2.4$ & $1.4 \pm 1.3$ & $3.9 \pm 2.8$ & $1.8 \pm 1.8$ & $2.8 \pm 1.9$ & $1.3 \pm 1$ & $2.4 \pm 1.9$ & $1.4 \pm 1.2$ & $2.0 \pm 1.4$ & $1.8 \pm 2.0$ \\
\hline Dyskinesia & & $1.7 \pm 2.9$ & & $1.5 \pm 2.6$ & & $1.5 \pm 2.1$ & & & & $1.1 \pm 0.3$ \\
\hline LEDD (mg) & & $1043 \pm 516$ & & $1180 \pm 548$ & & $1376 \pm 883^{*}$ & & & & $1054 \pm 488^{*}$ \\
\hline
\end{tabular}

Significant $P$ values:

Medical group off-medication vs surgical group off-medication on-stimulation: tremor $P=0.001$; akinesia: $P=0.015$.

Surgical group off-medication scores at baseline vs off-medication on-stimulation scores at 6 months: tremor $P=0.002 ;$ akinesia: $P=0.026$.

*One patient in surgical group had $8 \mathrm{mg}$ of trihexyphenidyl, both at baseline and at 6 months follow-up.

\section{Secondary outcomes}

Table 4 shows the values of selected items of the UPDRS-III and values for LEDD and UPDRS-IV (dyskinesia). Tremor improved markedly and akinesia moderately in the surgical group on-stimulation off-medication compared with the medical group off-medication. There were no differences between the groups in speech, axial symptoms, dyskinesia or in LEDD. When comparing the scores at 6 months with the scores at baseline in each group, total UPDRS-III scores as well as scores of subitems tremor and akinesia still showed good improvement on-medication versus off-medication in both groups.

When comparing UPDRS-III scores within the surgical group only, between on-stimulation and off-stimulation conditions at 6 months, there was a significant improvement mainly in tremor and moderately in akinesia.

Considering motor fluctuations, the majority of patients had mild fluctuations: at baseline, four patients in the surgical group and nine patients in the medical group reported predictable off periods (UPDRS-IV, item 36). Three patients in the surgical group and nine in the medical group reported unpredictable off periods (item 37). One patient reported sudden off periods in the medical group (item 38). The mean off-time score (item 39) was $0.9 \pm 0.9$ in the surgical group and $1.6 \pm 0.8$ in the medical group at baseline $(\mathrm{P}=\mathrm{NS})$. No statistically significant changes were seen within or between the groups at 6 months follow-up in any of the subitems 36-39.

\section{Adverse events}

In the surgical group, adverse events included a deep venous thrombosis in one patient 3 months after surgery that was treated successfully. No adverse events were encountered or reported in the medical group.

\section{Stimulation parameters}

Mean stimulation parameters at 6 months were $2.48 \pm 0.43$ V; $152.2 \pm 10.3 \mathrm{~Hz} ; 63.3 \pm 9.7 \mu \mathrm{s}$. Seven patients had monopolar and two bipolar stimulation. The most used contacts of the quadripolar electrode were the two middle contacts. No patient had the most proximal contact as a cathode. The active contacts (cathodes) for each individual patient with coordinates in relation to the midcommissural point are shown in figure 3. In relation to the visible posterior tail of the STN (pSTN), the active contacts were located at a mean of $1.9 \pm 1.1 \mathrm{~mm}$ medially, $0.6 \pm 0.5 \mathrm{~mm}$ posteriorly and $1.9 \pm 1.2 \mathrm{~mm}$ dorsally. The mean vectorial distance from the active contact to the pSTN was $3.1 \pm 0.8 \mathrm{~mm}$. In relation to the midcommissural point, the active contacts were located $2.5 \pm 1.2 \mathrm{~mm}$ below, $7.0 \pm 0.4 \mathrm{~mm}$ posterior and $11.6 \pm 1.1 \mathrm{~mm}$ lateral.

\section{DISCUSSION}

In this observer-blinded study on patients with advanced PD randomised to DBS in the $\mathrm{CZi}$ or to best medical management, it was shown that DBS in the cZi was safe and efficient. At 6 months, operated patients had 45\% improvement of UPDRS-III scores off-medication on-stimulation compared with their off-stimulation condition, and 41\% improvement compared with their baseline UPDRS-III scores. When compared with the non-surgical patients off-medication, the DBS-treated patients had $47 \%$ better UPDRS motor scores. There were no differences between the groups in the on-medication condition. The PDQ-39 summary index showed similar improvement in both groups at 6 months: the medical group improved by $24 \%$ and the DBS group by $36 \%$.

\section{Rationale for the study}

Two large reviews of published papers on DBS of the PSA and cZi for various movement disorders ${ }^{19}{ }^{20}$ have concurred in that this procedure seems to be quite efficient for tremor. One review hinted at the need for blinded evaluations, ${ }^{19}$ and the other review pointed to the need for quality-of-life assessments of patients. ${ }^{20}$ It is interesting to note that open-label studies that focused specifically on patients with PD undergoing cZi DBS reported a very positive effect on other symptoms than tremor: Carillo-Ruiz et al ${ }^{14}$ evaluated five patients and reported that the UPDRS-III was improved by $65 \%$, with a $90 \%$ improvement in tremor, $94 \%$ in rigidity and $75 \%$ in bradykinesia. In a non-randomised open-label sequential study, Plaha et $a l^{16}$ compared the effects of DBS in the STN (17 electrodes) and the cZi (27 electrodes) and reported that the best effect was achieved in the cZi with a reduction of contralateral UPDRS-III score by $76 \%$, tremor 93\%, rigidity $76 \%$ and bradykinesia $65 \%$. Nonetheless, according to the Movement Disorder Society task force on DBS for PD, only DBS targeting the STN or the GPi qualify as evidence-based procedures for PD. ${ }^{28}$ Hence, it was desirable 
Patient 1

Patient 2

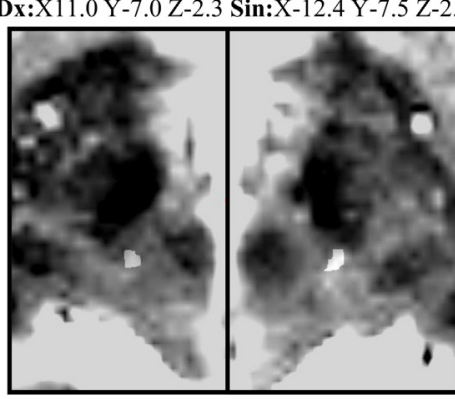

Patient 4

Dx:X12.1 Y-7.2 Z-2.9 Sin:X-13.0 Y-6.4 Z-1.8

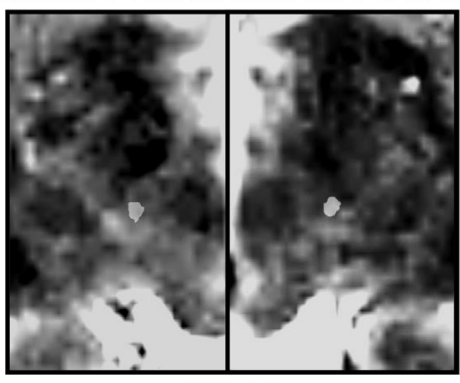

Patient 7

Dx:X11.5 Y-6.6 Z-3.4 Sin:X-12.3 Y-7.5 Z-4.8

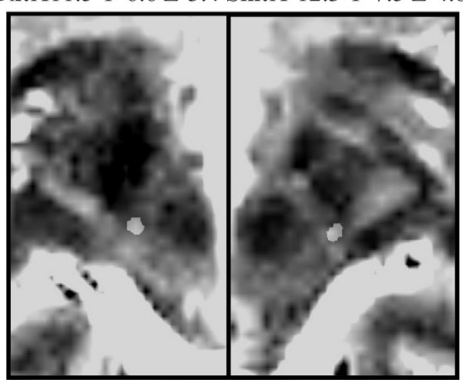

Sin:X-10.8 Y-7.3 Z-2.5

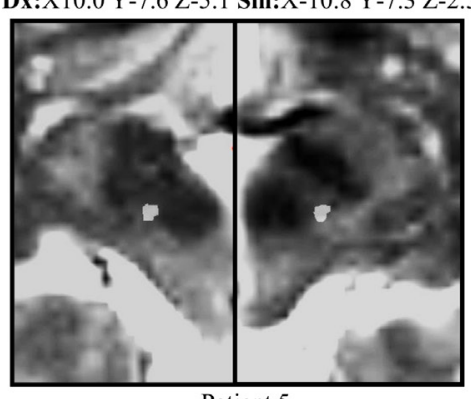

Patient 5

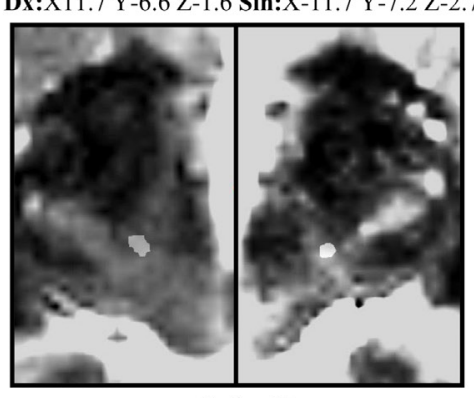

Patient 8

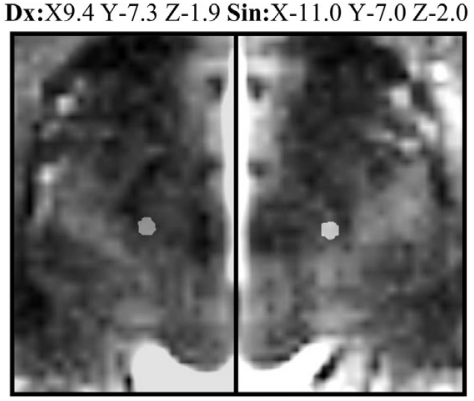

Patient 3

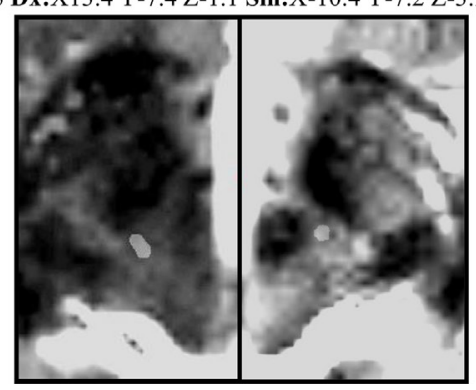

Patient 6

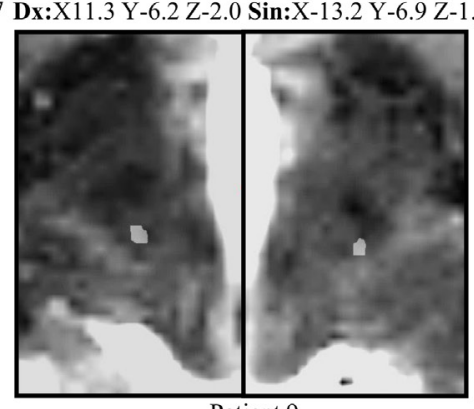

Patient 9

Dx:X11.6 Y-6.8 Z-1.2 Sin:X-12.5 Y-6.2 Z-2.6

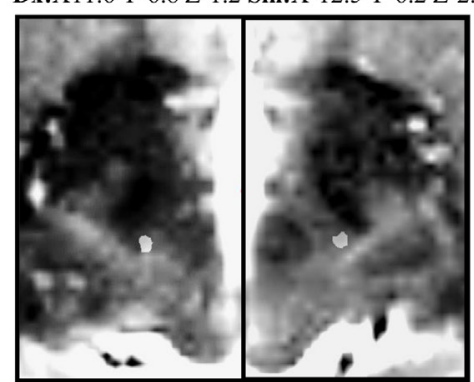

Figure 3 Fused axial CT-MRI scans at the level of the active contact bilaterally in all operated patients. Coordinates are given in relation to the midcommissural point.

to establish in an objective manner whether cZi DBS was efficient for PD and whether it was better than medication alone. It was also important to analyse its effect profile on the various symptoms of PD, and its impact on quality of life, in a similar way to what Deuschl et al did in their seminal publication from 2006 comparing STN DBS versus best medical management ${ }^{2}$ at 6-month follow-up. Therefore, we chose to include in this trial patients with PD who would otherwise qualify for STN DBS.

\section{Effect profile of cZi DBS on PD symptoms}

In analogy to most open-label reports, ${ }^{2} 15172729$ cZi DBS had a profound effect on tremor in our patients. There was a 92\% improvement in tremor scores when comparing DBS on versus baseline, and $93.4 \%$ improvement when comparing DBS on versus off at 6 months. Indeed, this is not surprising since high-frequency stimulation targeting the PSA and especially the area of the cZi will affect the cerebellothalamic fibres which are here concentrated in a small area, forming a bottleneck, before dispersing into the VIM thalamus. ${ }^{30-36}$ This would also explain the relatively modest level of energy needed in our patients to obtain a satisfactory effect on tremor, when stimulating this area (2.5 $\mathrm{V}$ in average).

The off-medication akinesia items of UPDRS-III showed 32\% improvement between DBS on and baseline, and 38\% improvement between DBS on and DBS off. This degree of improvement is less than what has been reported in other open-label non-blinded studies of DBS in PSA and $\mathrm{CZi}^{14}{ }^{16}$ and underscores the importance of blinded clinical evaluations in surgery for movement disorders, especially when investigating 'new' brain targets for DBS. Interestingly, the level of improvement in our study was not much different from what was shown in similar randomised studies of bilateral STN DBS, where Deuschl et $a l^{2}$ reported an improvement of $41 \%$ of UPDRS-III at 6 months in the German multicentre trial, and Williams et al $^{37}$ reported $36.2 \%$ improvement at 12 months in the British multicentre 'PD SURG' trial.

Finally, in our study, axial items and items related to speech and dyskinesia did not show any differences between groups, nor between on-stimulation and off-stimulation in the DBS group. Also, the LEDD were unchanged between groups and within groups, although there was a non-significant tendency for a reduction of LEDD in the surgical group. Hence, at variance with what is known from studies on STN DBS, our patients with cZi DBS remained on practically the same doses of dopaminergic medications at 6 months as before surgery.

\section{Strength and limitations of our study}

To the best of our knowledge, this is the first study on DBS in the $\mathrm{cZi}$ using a randomisation between a medical and a surgical group, and an observer-blinded evaluation. On the other hand, we are aware of the limited number of patients in this study: it 
was not straightforward to recruit participants because, since the patients had to be eligible for the established STN DBS procedures, several patients who were cleared for surgery and who were informed as per protocol that cZi DBS was an investigational target opted to have DBS in the more established STN target instead.

At baseline, disease duration was 4 years shorter on average in the surgical group than in the medical group, and the scores of UPDRS-III off-medication were lower. These differences were, however, not statistically significant, which could be due to the small sample size and/orlarge SD. This difference can also be explained by our non-stratified randomisation. Furthermore, at the 6-month follow-up, the differences in off-medication scores of UPDRS-III between groups had decreased and remained not significant.

Another limitation of our study is the relatively short follow-up of 6 months, but this was similar to the length of follow-up of the first randomised study comparing STN DBS to best medical management. ${ }^{2}$ Besides, it would be difficult to ethically justify withholding any surgery for longer than 6 months in patients with advanced disease who were eligible for surgery but who were in the medical arm.

\section{The place of cZi DBS in surgery for PD}

Our study confirmed a strong effect of DBS in the cZi on parkinsonian tremor and moderate effect on bradykinesia. Therefore, this brain target may be considered in tremor-dominant parkinsonian patients who for various reasons may not be eligible for bilateral STN or GPi DBS. DBS in cZi can also be performed unilaterally if needed. Concerning VIM versus cZi DBS, our experience ${ }^{27} 3839$ and that of others ${ }^{34-36}$ is that cZi DBS can be more efficient and more effective than VIM DBS. Additionally, as demonstrated in this study, DBS in cZi does have an effect, although moderate, on bradykinesia. Finally, compared with thalamic VIM DBS, cZi is also a target with more closely related anatomical structures that are visible on MRI, thereby allowing direct anatomical targeting as shown in figures 1 and 2.

In our practice of DBS, the cZi has now replaced the VIM both for selected patients with PD as well as patients with other tremor disorders. The fact that PD medications are not decreased lends this target to be used for unilateral DBS in patients with asymmetric parkinsonism.

\section{CONCLUSIONS}

In this observer-blinded study on patients with advanced PD randomised to DBS in the cZi or to best medical management, it was shown that DBS in the cZi was efficient. The powerful effect of cZi DBS on tremor was confirmed. There was a modest, although significant, improvement on akinesia but not of the same magnitude as the improvement reported following STN DBS. Future reports will provide data on longer-term follow-up. Also, further studies are needed in order to determine the role of cZi DBS in the therapeutic armamentarium for PD, especially in relation to STN DBS. What can be stated so far is that the cZi as a target for DBS in PD will be an addition to the existing established targets, which may increase the possibility to tailor the surgery to the needs of the individual patients.

Contributors $P B, L F, M H$ and RSP designed the research. RSP, G-MH, JL, AF, BH, JP, $\mathrm{LF}, \mathrm{MH}$ and $\mathrm{PB}$ conducted the study and acquired the data. RSP, G-MH, PB, MH and $\mathrm{JL}$ analysed and interpreted the data. RSP, PB and MH drafted the manuscript. All authors commented on and critically revised the manuscript. All authors approved the final version of the manuscript.
Funding This study was supported by a grant (ALF) from the University Hospital of Northern Sweden (Spjutspets), and the University of Umeå.

Competing interests $\mathrm{PB}$ is consultant for Medtronic and shareholder in Mithridaticum AB. JL has received honoraria for lectures from Lundbeck, AbbVie, IPSEN, Nordic InfuCare, netdoctor.se, and Medtronic. MH has received honoraria and travel expenses from Medtronic and Boston Scientific for speaking at meetings. $\mathrm{MH}$ is supported by the Parkinson Appeal UK, and the Monument Trust.

\section{Patient consent Obtained.}

Ethics approval Ethical Committee of Umeå University.

Provenance and peer review Not commissioned; externally peer reviewed.

Open access This is an open access article distributed in accordance with the Creative Commons Attribution Non Commercial (CC BY-NC 4.0) license, which permits others to distribute, remix, adapt, build upon this work non-commercially, and license their derivative works on different terms, provided the original work is properly cited and the use is non-commercial. See: http://creativecommons.org/ licenses/by-nc/4.0/

(c) Article author(s) (or their employer(s) unless otherwise stated in the text of the article) 2018. All rights reserved. No commercial use is permitted unless otherwise expressly granted.

\section{REFERENCES}

1 Weaver FM, Follett K, Stern M, et al. Bilateral deep brain stimulation vs best medical therapy for patients with advanced Parkinson disease: a randomized controlled trial. JAMA 2009:301:63-73.

2 Deuschl G, Schade-Brittinger C, Krack P, et al. A randomized trial of deep-brain stimulation for Parkinson's disease. N Engl J Med 2006;355:896-908.

3 Foltynie T, Hariz MI. Surgical management of Parkinson's disease. Expert Rev Neurother 2010:10:903-14.

4 Okun MS, Foote KD. Parkinson's disease DBS: what, when, who and why? The time has come to tailor DBS targets. Expert Rev Neurother 2010;10:1847-57.

5 Houdart R, Mamo H, Dondey M, et al. [Results of subthalamic coagulations in Parkinson's disease (apropos of 50 cases)]. Rev Neurol 1965;112:521-9.

6 Mundinger F. [Subthalamotomy for the treatment of extrapyramidal movement disorders]. Dtsch Med Wochenschr 1965;90:2002-7.

7 Story JL, French LA, Chou SN, et al. Experiences with subthalamic lesions in patients with movement disorders. Confin Neurol 1965;26:218-21.

8 Fager CA. Evaluation of thalamic and subthalamic surgical lesions in the alleviation of Parkinson's disease. J Neurosurg 1968;28:145-9.

9 Andy OJ, Jurko MF, SIAS FR. Subthalamotomy in treatment of parkinsonian tremor. J Neurosurg 1963;20:860-70.

10 Blomstedt P, Sandvik U, Fytagoridis A, et al. The posterior subthalamic area in the treatment of movement disorders: past, present, and future. Neurosurgery 2009;64:1029-38.

11 Gallay MN, Jeanmonod D, Liu J, et al. Human pallidothalamic and cerebellothalamic tracts: anatomical basis for functional stereotactic neurosurgery. Brain Struct Funct 2008;212:443-63.

12 Velasco F, Jiménez F, Pérez ML, et al. Electrical stimulation of the prelemniscal radiation in the treatment of Parkinson's disease: an old target revised with new techniques. Neurosurgery 2001;49:293-306.

13 Blomstedt P, Sandvik U, Tisch S. Deep brain stimulation in the posterior subthalamic area in the treatment of essential tremor. Mov Disord 2010;25:1350-6.

14 Carrillo-Ruiz JD, Velasco F, Jimènez F, et al. Bilateral electrical stimulation of prelemniscal radiations in the treatment of advanced Parkinson's disease. Neurosurgery 2008;62:347-59.

15 Kitagawa M, Murata J, Uesugi $\mathrm{H}$, et al. Two-year follow-up of chronic stimulation of the posterior subthalamic white matter for tremor-dominant Parkinson's disease. Neurosurgery 2005;56:281-9.

16 Plaha P, Ben-Shlomo Y, Patel NK, et al. Stimulation of the caudal zona incerta is superior to stimulation of the subthalamic nucleus in improving contralateral parkinsonism. Brain 2006;129:1732-47

17 Plaha P, Khan S, Gill SS. Bilateral stimulation of the caudal zona incerta nucleus for tremor control. J Neurol Neurosurg Psychiatry 2008;79:504-13.

18 Blomstedt P, Fytagoridis A, Tisch S. Deep brain stimulation of the posterior subthalamic area in the treatment of tremor. Acta Neurochir 2009;151:31-6.

19 Xie T, Bernard J, Warnke P. Post subthalamic area deep brain stimulation for tremors: a mini-review. Trans/ Neurodegener 2012;1:20.

20 Ramirez-Zamora A, Smith H, Kumar V, et al. Evolving concepts in posterior subthalamic area deep brain stimulation for treatment of tremor: surgical neuroanatomy and practical considerations. Stereotact Funct Neurosurg 2016;94:283-97

21 Voon V, Krack $P$, Lang $A E$, et al. A multicentre study on suicide outcomes following subthalamic stimulation for Parkinson's disease. Brain 2008;131:2720-8.

22 Kulisevsky J, Berthier ML, Gironell A, et al. Mania following deep brain stimulation for Parkinson's disease. Neurology 2002;59:1421-4. 
23 Hindle Fisher I, Pall HS, Mitchell RD, et al. Apathy in patients with Parkinson's disease following deep brain stimulation of the subthalamic nucleus. CNS Spectr 2016;21:258-64.

24 Bejjani BP, Damier P, Arnulf I, et al. Transient acute depression induced by highfrequency deep-brain stimulation. N Engl J Med 1999;340:1476-80.

25 Blomstedt $\mathrm{P}$, Hariz MI, Lees $\mathrm{A}$, et al. Acute severe depression induced by intraoperative stimulation of the substantia nigra: a case report. Parkinsonism Relat Disord 2008:14:253-6.

26 Gibb WR, Lees AJ. The relevance of the Lewy body to the pathogenesis of idiopathic Parkinson's disease. J Neurol Neurosurg Psychiatry 1988;51:745-52.

27 Blomstedt P, Fytagoridis A, Åström M, et al. Unilateral caudal zona incerta deep brain stimulation for Parkinsonian tremor. Parkinsonism Relat Disord 2012;18:1062-6.

28 Fox SH, Katzenschlager R, Lim SY, et al. The movement disorder society evidencebased medicine review update: treatments for the motor symptoms of Parkinson's disease. Mov Disord 2011;26(Suppl 3):S2-41.

29 Castro G, Carrillo-Ruiz JD, Salcido V, et al. Optimizing prelemniscal radiations as a target for motor symptoms in Parkinson's disease treatment. Stereotact Funct Neurosurg 2015;93:282-91.

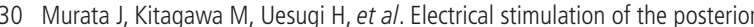
subthalamic area for the treatment of intractable proximal tremor. J Neurosurg 2003;99:708-15.

31 Mitrofanis J, deFonseka R. Organisation of connections between the zona incerta and the interposed nucleus. Anat Embryol 2001;204:153-9.
32 Vitek JL, Ashe J, DeLong MR, et al. Physiologic properties and somatotopic organization of the primate motor thalamus. J Neurophysiol 1994;71:1498-513.

33 Plaha P, Patel NK, Gill SS. Stimulation of the subthalamic region for essential tremor. J Neurosurg 2004;101:48-54.

34 Hamel W, Herzog J, Kopper F, et al. Deep brain stimulation in the subthalamic area is more effective than nucleus ventralis intermedius stimulation for bilateral intention tremor. Acta Neurochir 2007;149:749-58.

35 Herzog J, Hamel W, Wenzelburger R, et al. Kinematic analysis of thalamic versus subthalamic neurostimulation in postural and intention tremor. Brain 2007;130:1608-25.

36 Barbe MT, Liebhart L, Runge M, et al. Deep brain stimulation of the ventral intermediate nucleus in patients with essential tremor: stimulation below intercommissural line is more efficient but equally effective as stimulation above. Exp Neurol 2011;230:131-7.

37 Williams A, Gill S, Varma T, et al. Deep brain stimulation plus best medical therapy versus best medical therapy alone for advanced Parkinson's disease (PD SURG trial): a randomised, open-label trial. Lancet Neurol 2010;9:581-91.

38 Hariz MI, Shamsgovara P, Johansson F, et al. Tolerance and tremor rebound following long-term chronic thalamic stimulation for Parkinsonian and essential tremor. Stereotact Funct Neurosurg 1999;72:208-18.

39 Sandvik U, Koskinen LO, Lundquist A, et al. Thalamic and subthalamic deep brain stimulation for essential tremor: where is the optimal target? Neurosurgery 2012;70:840-5. 